\title{
The Eschenmoser coupling reaction under continuous-flow conditions
}

\author{
Sukhdeep Singh, J. Michael Köhler, Andreas Schober \\ and G. Alexander Groß*
}

Open Access

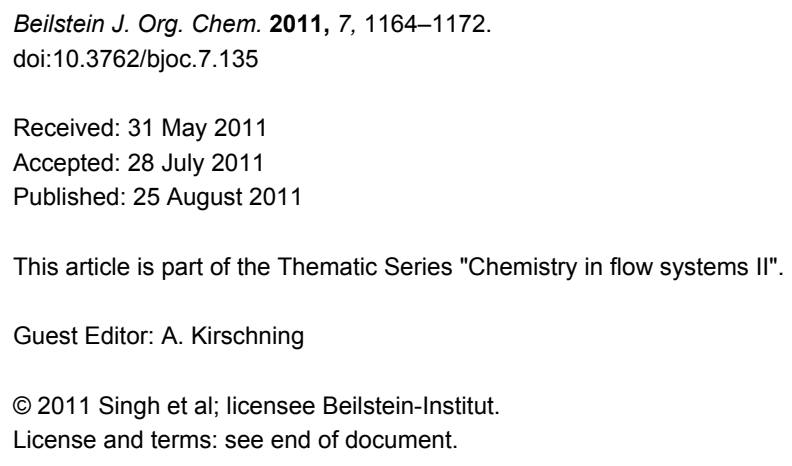

\begin{abstract}
The Eschenmoser coupling is a useful carbon-carbon bond forming reaction which has been used in various different synthesis strategies. The reaction proceeds smoothly if S-alkylated ternary thioamides or thiolactames are used. In the case of S-alkylated secondary thioamides or thiolactames, the Eschenmoser coupling needs prolonged reaction times and elevated temperatures to deliver valuable yields. We have used a flow chemistry system to promote the Eschenmoser coupling under enhanced reaction conditions in order to convert the demanding precursors such as S-alkylated secondary thioamides and thiolactames in an efficient way. Under pressurized reaction conditions at about $220{ }^{\circ} \mathrm{C}$, the desired Eschenmoser coupling products were obtained within $70 \mathrm{~s}$ residence time. The reaction kinetics was investigated and 15 examples of different building block combinations are given.
\end{abstract}

\section{Introduction}

The Eschenmoser coupling $[1,2]$ is a reaction method that yields $\beta$-enaminocarbonyl derivatives of type 4 by the elimination of sulfur (sulfide contraction) from an episulfide intermediate (Scheme 1). The reaction was described for the first time by Knott in 1955 [3] and became prominent later on when it was applied to the total synthesis of vitamin B12 by Eschenmoser [4]. Since these early days the Eschenmoser coupling has been applied many times as a useful reaction step in a variety of synthesis strategies. Different natural products, such as diplodialid macrolactone- [5], sedamine alkaloid- [6], sparteine- [7], mersicarpine- [8], batzelladine- [9], fuligocandin- [10] and vitamin B12-derivatives [11] were prepared with the aid of sulfide contraction steps. Pharmaceutically important substances, such as methylphenidat [12] or the marine neurotoxin hemibreve- 


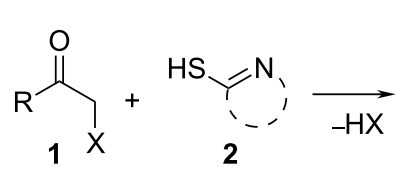<smiles>[R]C(=O)CSC1=NCCC1</smiles><smiles>[R]C(=O)C=C1NCCN1</smiles>

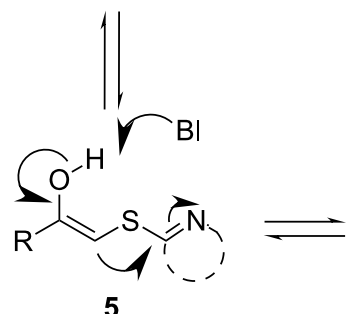

$-\mathrm{s}$

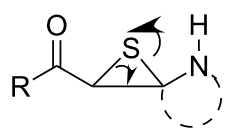

6

Scheme 1: Eschenmoser coupling reaction with secondary S-alkylated thioamide derivatives of type 3.

toxin [13], were prepared by utilization of the Eschenmoser coupling reaction. Recently, we explored the Eschenmoser coupling in order to produce Biginelli type dihydropyrimidine (DHPM) derivatives as screening candidates for pharmaceutical and crop science research [14]. Moreover, the Eschenmoser coupling is quite a valuable, metal free, carbon-carbon bond forming reaction.

The necessary starting materials of type 3 can be prepared by S-alkylation of secondary thioamide or thiolactame building blocks of type 2 with $\alpha$-bromoketones 1 (X $=\mathrm{Br})$. The carbon-carbon coupling occurs between these building blocks as shown in Scheme 1. Due to the ease of accessibility of the building blocks and the usually high-yielding S-alkylation step, the Eschenmoser coupling is an interesting reaction for diversity-oriented combinatorial synthesis [15-17].

The new carbon-carbon bond formation occurs during the construction of the episulfide intermediate $\mathbf{6}$, which requires base catalysis $(\mathrm{B} \mid)$. The sulfur extraction from the episulfides 6 or 8 yields the desired $\beta$-enaminocarbonyl derivative 4 or 9 . However, the detailed reaction mechanism for the sulfur extraction step has not yet been fully proven and obviously depends on the applied reaction conditions. Experimental observations show that the reaction can take place without the addition of a thiophilic agent or base. This is a strong hint that the sulfide extraction is of an intramolecular pericylic nature, as proposed in Scheme 1. In the presence of a thiophilic agent, the mechanism of extraction via intermediate ionic states also seems plausible. Nevertheless, the Eschenmoser coupling reaction requires the addition of a base and a thiophilic agent in the most cases. Here, triphenylphosphine- or trialkyl-phosphite-derivatives are usually employed to promote the sulfide contraction with valuable yield and selectivity. Eschenmoser himself made use of bi-functional reagents with dual thiophile and basic properties in one molecule [2]. With regards to experimental considera- tions, the thiophilic reagent must be carefully chosen in order to overcome the difficulties in the separation of the desired product from phosphine sulfide byproducts.

S-Alkylated ternary thioamides of type 7 usually undergo the episulfide formation and subsequent sulfide contraction smoothly (Scheme 2). This is due to the strong electron accepting nature of the thioiminium intermediate 8. Unfortunately, the S-alkylation is sometimes difficult in these cases [6]. In the case of secondary thiolactames of type $\mathbf{5}$, sulfide contraction takes place as well, but prolonged reaction time and increased reaction temperature are necessary in most cases. In contrast to this, secondary thioamide derivatives of type $\mathbf{5}$ undergo the S-alkylation with $\alpha$-bromoketones more readily than ternary thioamides. However, the specific reactivity of both reaction steps obviously depends on the building blocks used and their specific reactivity. For the present work we chose only commercially available $\alpha$-bromoketone building blocks and secondary thiolactame and thioamide derivatives. To improve the long reaction times we investigated the Eschenmoser coupling under process intensification conditions. Therefore, reaction temperatures far beyond the solvent boiling point were applied under pressurized flow conditions for a minimum residence time. For small scale synthesis flow chemistry is of great advantage to realize these conditions on the laboratory bench safely [18].

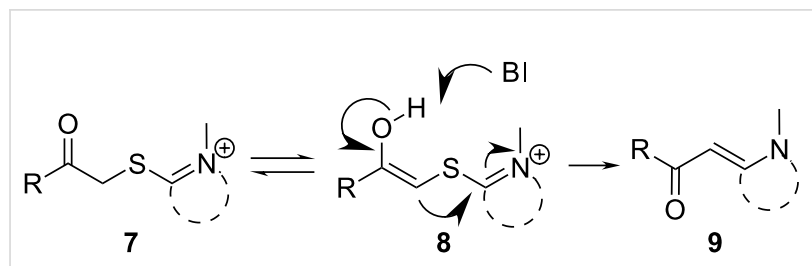

Scheme 2: Eschenmoser coupling sequence of S-alkylated ternary thioamides of type 7 . 


\section{Results and Discussion}

For the reaction optimization we have focused on a straightforward procedure that finally prevents the isolation of the S-alkylated intermediates 3 and avoids a change of the reaction solvent for the subsequent step. To establish the best conditions we investigated the reaction of 2-bromo-1-phenylethanone (1a) with 2-mercapto-6-methyl-pyrimidin-4-ol (2a) in detail. Therefore, 2a was dissolved in different solvents and 2 equiv $\mathrm{NEt}_{3}$ were added. After $10 \mathrm{~min}$, the dissolved $\alpha$-bromoketone $1 \mathrm{a}$ was added dropwise. A concentration of about $0.1 \mathrm{~mol} / \mathrm{L}$ was achieved in this way. The reaction solutions were sonicated for about $1 / 2 \mathrm{~h}$. Subsequently, the $\mathrm{HNEt}_{3} \mathrm{Br}$ precipitates were filtered off. The precipitation and filtration was done with care to prevent malfunctions of the feeding pumps. In the case of chloroform as solvent no precipitation took place, but the LC-MS analysis indicated the S-alkylation clearly, with a purity of about $89 \%$. To all filtered solutions 1.25 equiv of triisopropylphosphite (TIP) were added as thiophilic agent before the reaction solutions were fed through the flow chemistry setup. A flow rate of $1000 \mu \mathrm{L} / \min$ (RT: $55 \mathrm{~s}$ ) was adjusted and the reaction temperature was increased stepwise from 120 to $240{ }^{\circ} \mathrm{C}$. The backpressure regulator was set for all experiments to about 100 bar. The received reaction products were collected and analyzed by LC-MS analysis. In the case of THF as solvent, precipitation took place in the capillary and the backpressure regulator became blocked. In case of chloroform, the reaction solution turned black, and oily polymeric products were formed when the reaction temperature exceeded $150{ }^{\circ} \mathrm{C}$. As a result, only traces of the desired product 4 aa were observed by LC-MS analysis, along with multiple side products. The side products were not investigated in greater detail because of the complexity of the mixture. In the case of ethanol as solvent, the solubility of the starting materials and S-alkylated intermediate 3aa was poor and the solutes tended to precipitate. However, anhydrous 1,4-dioxane was found to be the best solvent for both reaction steps. Conversions, with anhydrous 1,4-dioxane as solvent, are shown in Figure 1. Only poor conversion was observed up to $160{ }^{\circ} \mathrm{C}$. The maximum conversion of about $98 \%$ was observed at $220{ }^{\circ} \mathrm{C}$, with a flow rate of $250 \mu \mathrm{L} / \mathrm{min}$. At reaction temperatures beyond $220{ }^{\circ} \mathrm{C}$ the selectivity of the reaction decreased dramatically and some unidentified decomposition products appeared. Nevertheless, the increase of the flow rate, to about 500 or $750 \mu \mathrm{L} / \mathrm{min}$ at $230{ }^{\circ} \mathrm{C}$, led to comparable good yields and high reaction selectivities. In the case of higher flow rate, of $1000 \mu \mathrm{L} / \mathrm{min}$ and beyond, and the corresponding shorter reaction time, a decreased conversion was observed due to the slow reaction kinetics (Figure 1).

The flow chemistry technique allows the determination of reaction kinetics data in a fast and efficient way, even under pressurized process-identifying conditions. We investigated the kinetics of the sulfide contraction of 3aa to $4 \mathbf{a a}$ in detail. For this purpose, a $0.1 \mathrm{~mol} / \mathrm{L}$ reaction solution was fed through the reaction system at different flow rates and reaction tempera-

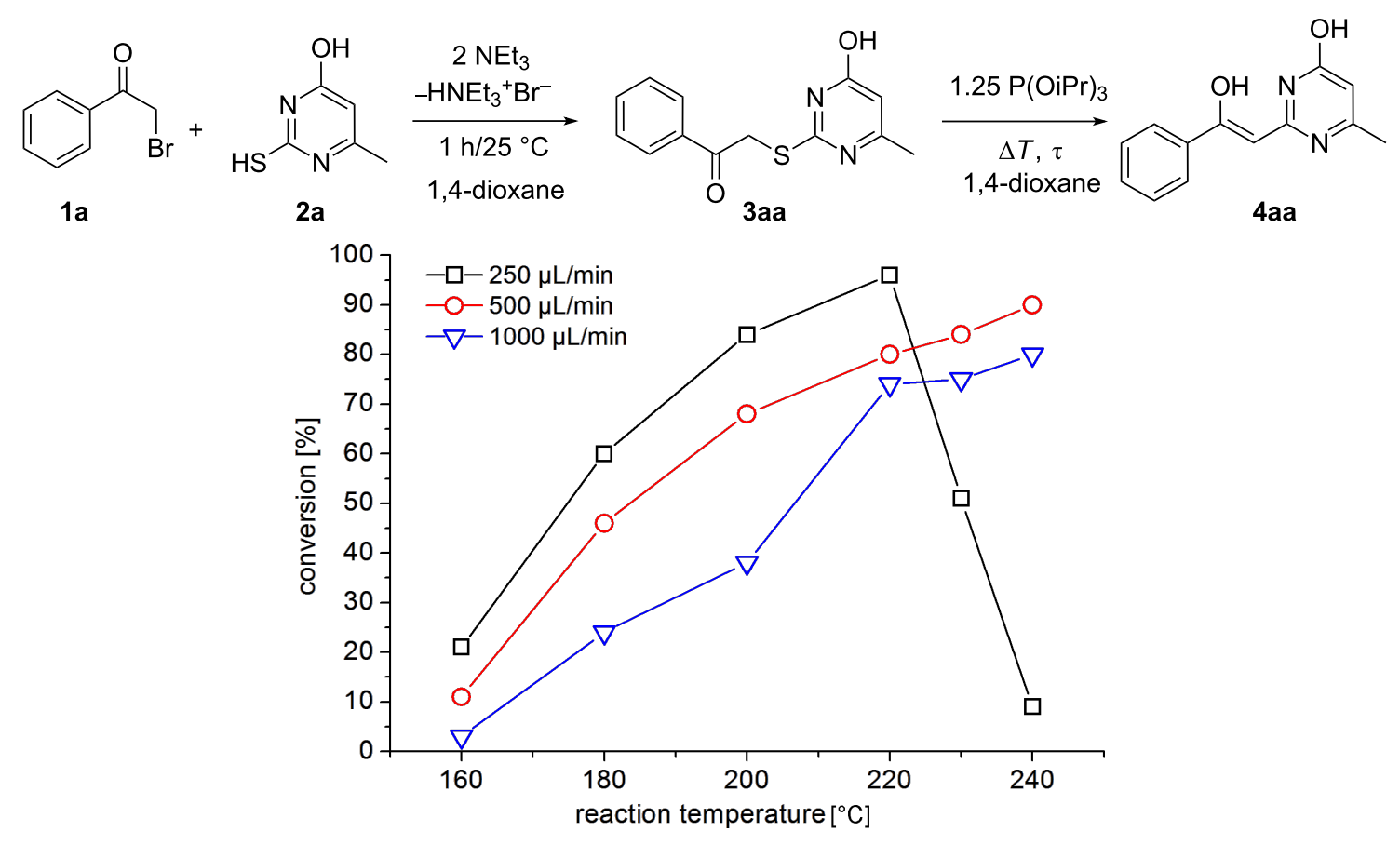

Figure 1: Conversion of 3aa to 4 aa under different flow conditions. 
tures. After each change in the reaction parameters the reaction system was allowed to reach a steady state before a sample was taken. After a change of the flow rate, samples were taken only after a minimum waiting period of about three times the residence time to achieve system equilibration.

In order to compare the reaction performance of the flow reaction with the conventional technique, the same reaction was carried out under batch conditions. The resulting product solutions were analyzed by LC-MS and isolated by the standard precipitation/recrystallization procedure. No conversion was observed in the batch reaction at ambient temperature within $48 \mathrm{~h}$. Even heating up to the boiling point of 1,4-dioxane (101 ${ }^{\circ} \mathrm{C}$ ) for about $1 \mathrm{~h}$ did not furnish the desired product in any significant amount. However, after refluxing overnight a yield of at least $43 \%$ 4aa was isolated.

The investigated Eschenmoser coupling under flow conditions was found to be a first order reaction. Rate constants were calculated using the first order kinetic Equation 1, where $c$ $[\mathrm{mol} / \mathrm{L}]$ is the concentration of the product $\mathbf{4 a a}$ at the reaction temperature $T[\mathrm{~K}]$ after the reaction solution was passed through the heater with the residence time $\tau$ [s]. The resulting kinetic plot is shown in Figure 2 (left). The determined rate constants were used to prepare an Arrhenius plot and for calculation of the activation energy from Equation 2. The resulting Arrhenius plot is shown in Figure 2 (right). An activation energy $E_{\mathrm{A}}$ of about $91 \mathrm{~kJ} / \mathrm{mol}$, and a frequency factor $A$ of about $3.73 \times 10^{9} \mathrm{~s}^{-1}$, were found. This activation energy is obviously the reason for the slow reaction kinetics at moderate temperature. Hence, the application of pressurized high temperature conditions should lead to significant reaction intensification.

$$
\begin{gathered}
\ln \left(\frac{c}{c_{0}}\right)=-k(T) \cdot \tau \\
k(T)=A \cdot e^{-\frac{E_{a}}{R \cdot T}}
\end{gathered}
$$

For the analysis of the reaction kinetics the following workflow was necessary: 1) Variation of the reaction parameters, 2) probe sampling, 3) HPLC analysis and 4) reaction kinetics calculations for six different temperatures at five different flow rates. The determination of the complete reaction kinetics data was complete within $6 \mathrm{~h}$. A comparable analysis by a sequential batch technique would take approximately 1 week. Hence, flow chemistry is well suited to efficiently determine the kinetics for reactions under unconventional reaction conditions.

To explore the scope of the sulfide-contraction reaction we varied both building blocks in a systematic manner under the following reaction conditions: $0.1 \mathrm{M}$ reaction solution in anhydrous 1,4-dioxane, 2 equiv $\mathrm{NEt}_{3}, 1.25$ equiv TIP at $220{ }^{\circ} \mathrm{C}$ and $250-750 \mu \mathrm{L} / \mathrm{min}$ flow rate. First, we varied the thiolactame building blocks 2 and kept the $\alpha$-bromoketone 1a constant. In Table 1, the investigated building blocks $\mathbf{2 a}-\mathbf{2} \mathbf{i}$ and the isolated yields are shown. For the product workup, $100 \%$ volume of water and $1 \% 1 \mathrm{M} \mathrm{HCl}$ were added to the product solution. If precipitation took place after addition, the residues were filtered. If no precipitation took place, then the reaction solution was extracted with ethyl acetate. The organic phase was separated and dried over $\mathrm{MgSO}_{4}$ and the ethyl acetate was subsequently removed by evaporation. The resulting extracts or precipitates were recrystallized from a solvent mixture of hot methanol/dichloromethane. However, the crystallization process
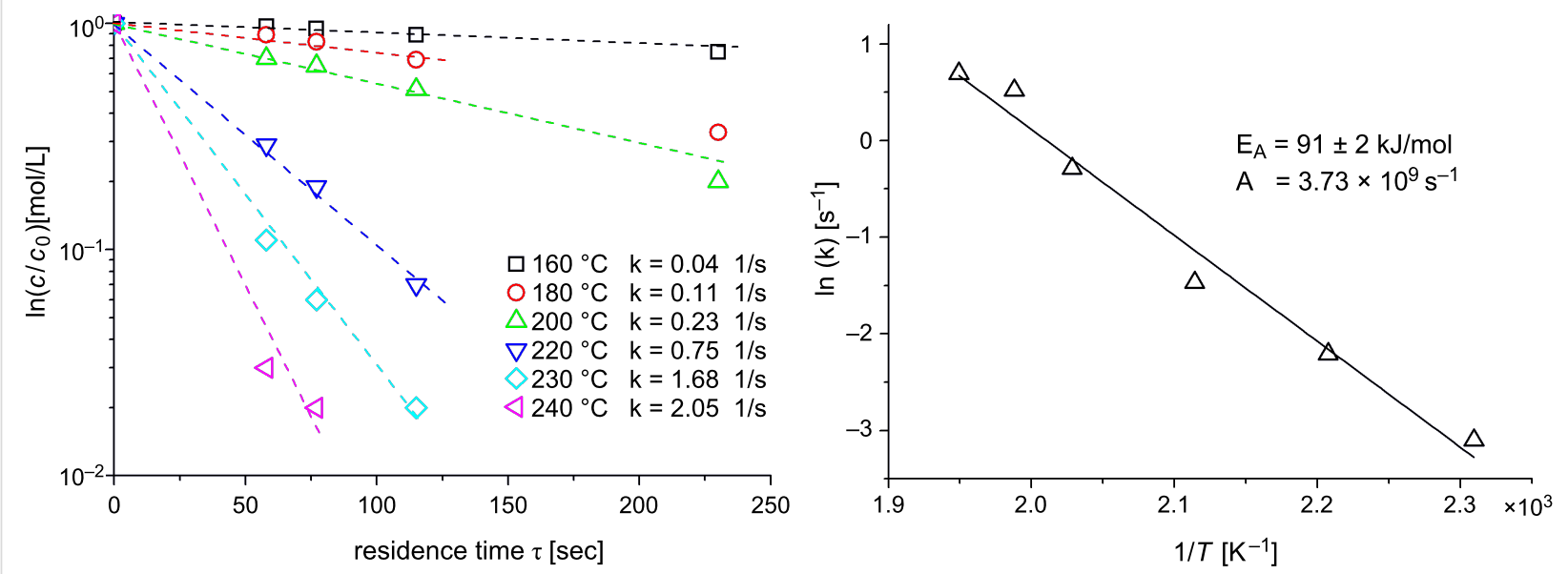

Figure 2: Reaction kinetics analysis. Left: Rate constants with $0.1 \mathrm{M}$ reaction solution. Right: Arrhenius-plot for the determined rate constants. 
Table 1: Product and yields received for different thio-substrates 2.

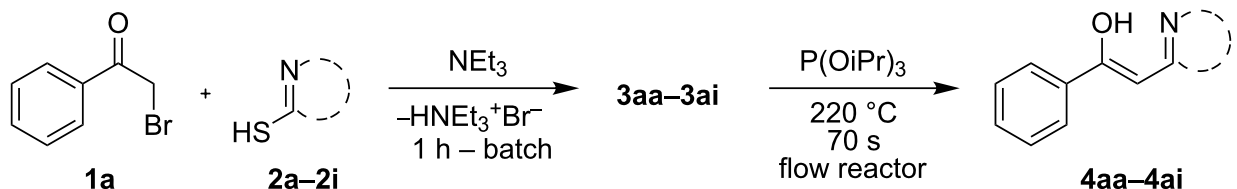

2a-2i

$2 a$<smiles>Cc1cc(O)nc(S)n1</smiles>

2b<smiles>Cc1nc(S)nc(O)c1C</smiles>

2c<smiles>Oc1nc(S)nc2ccccc12</smiles>

2d<smiles>Cc1nnc(S)nc1O</smiles>

$2 e$<smiles>CCOC(=O)C1=C(C)NC(S)=NC1c1ccc(OC)cc1</smiles>

$2 f$<smiles>CCOC(=O)C1=C(C)NC(S)=NC1c1ccccc1</smiles>

$2 \mathrm{~g}$<smiles>O=C1NC(=S)NC1(c1ccccc1)c1ccccc1</smiles>

$4 a a$<smiles>Cc1cc(O)nc(/C=C(\O)c2ccccc2)n1</smiles>

4ab<smiles>Cc1nc(/C=C(\O)c2ccccc2)nc(O)c1C</smiles>

$4 a c$<smiles>O/C(=C\c1nc(O)c2ccccc2n1)c1ccccc1</smiles>

4ad<smiles>Cc1nnc(/C=C(\O)c2ccccc2)nc1O</smiles>

$4 a e$<smiles>CCOC(=O)C1=C(C)NC(/C=C(\O)c2ccccc2)=NC1c1ccc(OC)cc1</smiles>

4af<smiles>CCOC(=O)C1=C(C)NC(/C=C(\O)c2ccccc2)=NC1c1ccccc1</smiles>

4ag<smiles>O=C1N=C(/C=C(\O)c2ccccc2)NC1(c1ccccc1)c1ccccc1</smiles> 
Table 1: Product and yields received for different thio-substrates 2. (continued)

$2 \mathrm{~h}$<smiles>Sc1nc2ccccc2[nH]1</smiles>

2i

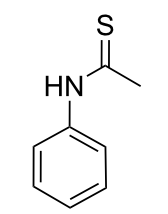

$4 a h$<smiles>O/C(=C\c1nc2ccccc2[nH]1)c1ccccc1</smiles>

4ai

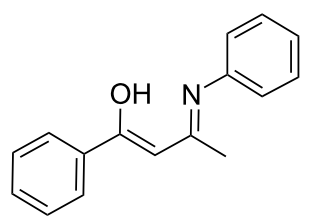

58

alsolated yields; ${ }^{b}$ Decomposition during sulfide contraction; ${ }^{\mathrm{c}}$ No product precipitation or extraction of 4 ah was possible; only 3 ah was isolated in smaller amount.

was not optimized and there is scope to increase yields further. The desired products 3 were received in synthetically useful yields in almost all cases. The NMR spectra of the resulting products $\mathbf{4}$ mainly comprise two sets of signals with varying intensities (details can be found in the Supporting Information File 1). This is due to the two different possibilities for the intramolecular H-bond formation caused by the keto-enol tautomerism of 4 . In this scheme, the reaction of 2-mercaptopyrimidin-4-ol derivatives $\mathbf{2 a}, \mathbf{2 b}$ and $\mathbf{2 c}$ proceeded smoothly with high yields. 6-Aza-2-thiothymine (2d) was quantitatively S-alkylated but decomposed during the sulfide-contraction step and subsequent workup. Therefore, we could not isolate the desired product $\mathbf{4 a d}$. The reaction of dihydropyrimidine substrates $\mathbf{2 e}$ and $\mathbf{2 f}$ proceeded in a facile way, and the desired products 3ae and 3af were isolated in good yields. Even the mercapto hydantoin derivative $\mathbf{2 g}$ underwent the two step reaction smoothly and yielded the desired product $\mathbf{4 a g}$ in good quantity. In case of the $1 H$-benzoimidazole-2-thiol $(\mathbf{2} \mathbf{h})$, the selective S-alkylation failed. The utilized alkylation conditions favor the bis-alkylation, and hence the S-alkylated product was produced in less than 10\% yield (HPLC-peak area). LC-MS analysis after the sulfide contraction of this mixture showed that the bis-S-alkylated intermediate was only partially converted. Unfortunately, we could not isolate the product from the complex reaction mixture by the standard precipitation and extraction/crystallization processes. The 2-thiobenzimidazol (2h) underwent the S-alkylation as well as the sulfide contraction smoothly and showed good conversion to the desired product 3ah. However, the applied precipitation technique yielded only a trace amount of the unconverted intermediate $\mathbf{3 a h}$. $\mathrm{N}$-Phenylthioacetamide (2i) was additionally chosen as a noncyclic secondary thioamide example. The desired product 4ai was received in excellent yield.

Various $\alpha$-bromoketone building blocks, as shown in Table 2, were investigated in combination with the thiolactame $\mathbf{2 a}$. We choose only aromatic bromoketones because of their known good reactivity and selectivity for the sulfide contraction [1]. Recently, we investigated the reaction of dihydropyrimidine derivatives of type $\mathbf{1 1}$ with different aliphatic ketones $\mathbf{1 0}$ under batch conditions. In that case the S-alkylated substrates $\mathbf{1 2}$ did not undergo the sulfide contraction but rather the thiazole formation to 13 took place exclusively (Scheme 3) [19]. The thiazol formation by condensation is a well known side reaction of the sulfide contraction. Nevertheless, and in contrast to the batch reaction, no thiazole formation was observed under the investigated flow conditions.

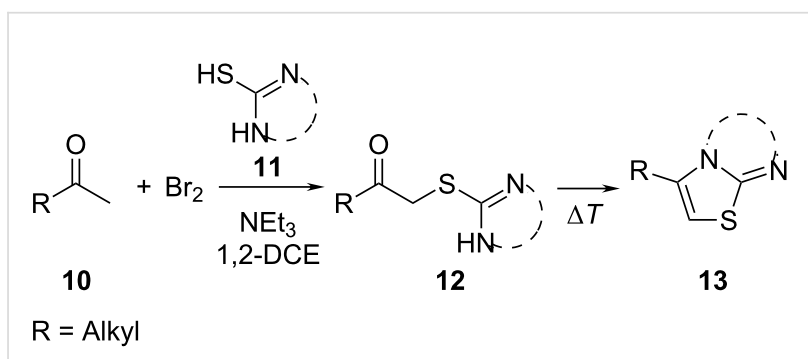

Scheme 3: Exclusive formation of thiazol 13 with dihydropyrimidine derivatives 11 take place in the case of aliphatic ketones 10 [19].

The influence of the bromoketone building blocks 1a-1g on the flow reaction performance was investigated (Table 2). 2-Mercapto-6-methylpyrimidin-4-ol (2a) was chosen as the reaction partner for all bromoketones $\mathbf{1 a}-\mathbf{1 g}$. High yields and reaction selectivities were observed in all cases at the investigated temperature of $220^{\circ} \mathrm{C}$. The influence of the different aromatic bromoketones on the reaction seems to be negligible. The electron accepting nature of the thio-substrate seems to have the dominating effect on the reaction performance. However, all investigated combinations of the building blocks furnished the desired products in good yields within residence times of about $70 \mathrm{~s}$. 


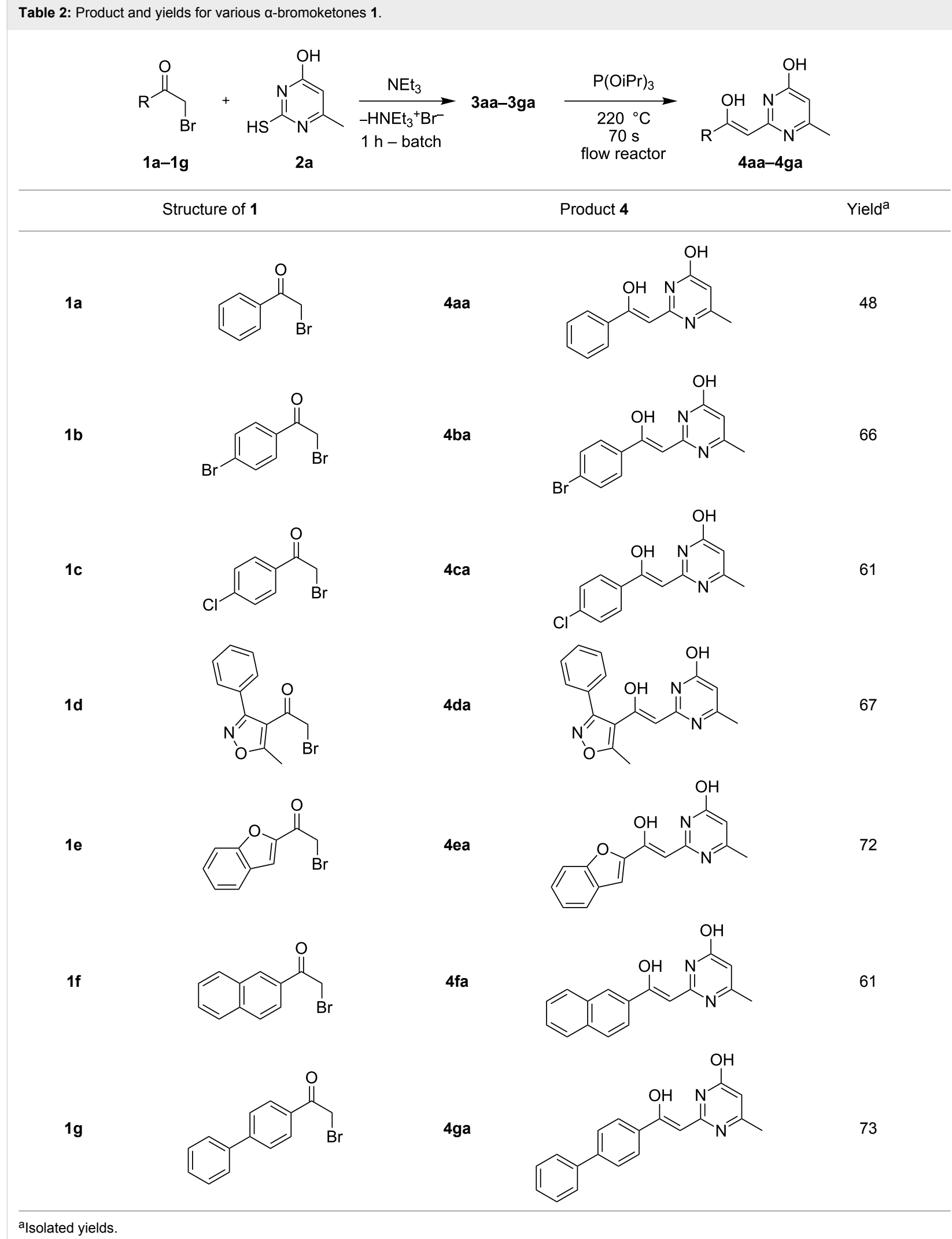




\section{Conclusion}

We found that Eschenmoser coupling can be performed at a reaction temperature of $220^{\circ} \mathrm{C}$ with residence times of about 70 s. 1,4-Dioxane was found to be the most effective solvent. The reaction kinetics was determined in a fast and efficient way for $0.1 \mathrm{M}$ reaction concentration. As a result, the flow chemistry technique enabled the significant intensification of the reaction kinetics, by application of enhanced process conditions on the laboratory bench, in a safe and efficient way. The intensification potential was investigated for demanding secondary thiolactames of type $\mathbf{5}$, and exemplary combinations of building blocks, as shown in Table 1 and Table 2, were examined. In almost all investigated cases the desired products were received in synthetically useful yields.

\section{Experimental}

\section{Flow chemistry system}

A flow chemistry assembly consisting of two feeding pumps, a capillary reactor, a heat controller and a backpressure regulator was used, as shown in Figure 3. The capillary reactor consists of a $2.1 \mathrm{~m}$ long steel capillary with $0.75 \mathrm{~mm}$ inner diameter $(\sim 930 \mu \mathrm{L})$, which was wrapped around a brass cylinder with an integrated heat-rod (hotset, HHP $200 \mathrm{~W}, 8 \times 80 \mathrm{~mm}$ ) and jacketed by a thermally isolated brass cover (Figure 4 ). Thermal control was achieved by using a commercial process controller (EMKO process controller ESM-4450) with adapted PT-100 temperature sensor. For pressure control a manual needle valve (micro splitter, Upchurch) was integrated at the capillary outlet. About $20 \mathrm{~cm}$ of the capillary between the heating cylinder and the backpressure regulator was tempered by a water chiller to ambient temperature. Feeds were delivered by the help of two double piston HPLC-pumps (Shimadzu LC-9A, double inlet check valves). The first pump was used to feed the reaction solutions and the second pump was used to feed pure solvent for purging and dilution. The maximum operational parameters for the complete flow chemistry setup were proven up to $250{ }^{\circ} \mathrm{C}$ and 200 bar. Residence times $(\tau)$ between $40 \mathrm{~min}$ and $60 \mathrm{~s}$ were achieved by adjusting the flow rate from 20 to $2000 \mu \mathrm{L} / \mathrm{min}$.

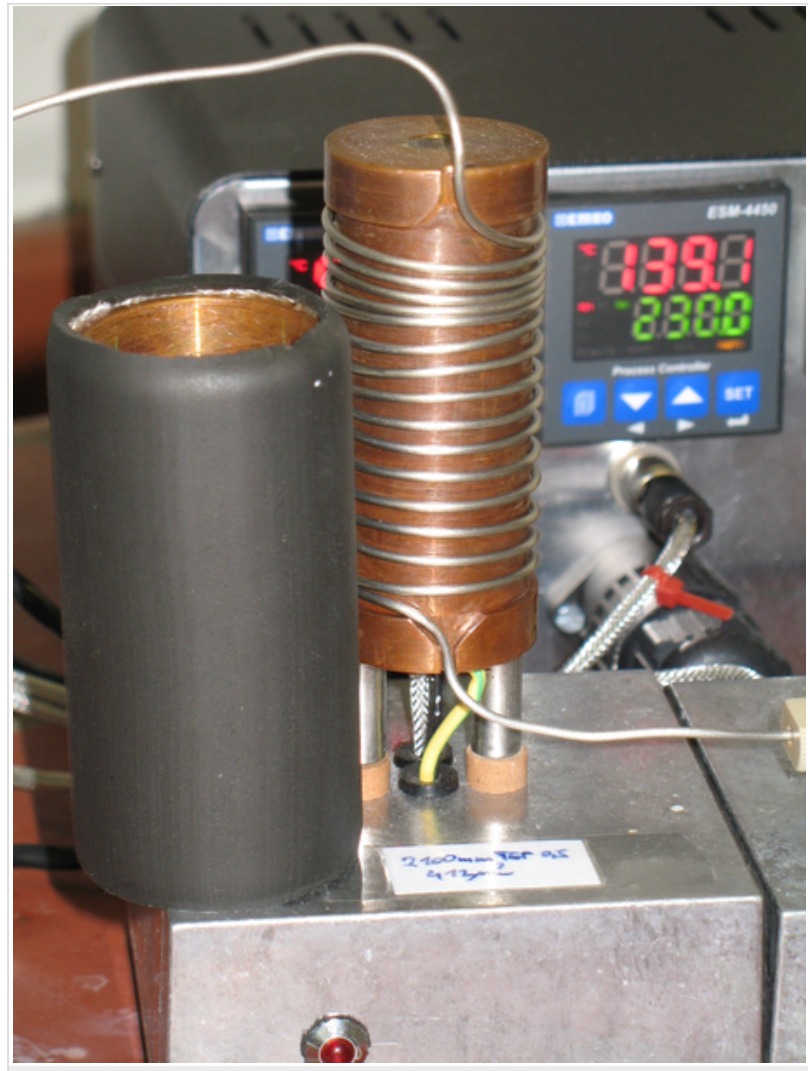

Figure 4: Capillary reactor with jacketed cover removed, and the process controller.

\section{Supporting Information}

\section{Supporting Information File 1}

General procedures, analytical and spectral data. [http://www.beilstein-journals.org/bjoc/content/ supplementary/1860-5397-7-135-S1.pdf]

\section{Acknowledgments}

The support from K. Risch and S. Günther for compound analysis is gratefully acknowledged. The authors thank

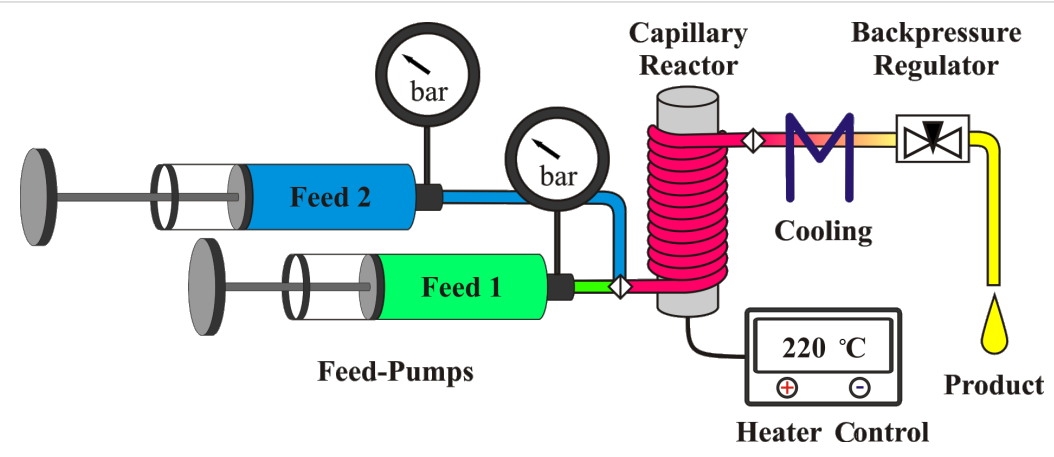

Figure 3: Flow chemistry setup scheme. 
S. Schneider for the support during the development of the reaction setup. This work was partially supported by different BMBF projects: OPTIMI (FKZ: 16SV3701), BioMepSens (FKZ: 03ZIK062) and Mikrokunststoffformen (FKZ: 03ZIK465).

\section{References}

1. Shiosaki, K. The Eschenmoser Coupling Reaction. In Comprehensive Organic Synthesis; Trost, B. M., Ed.; Pergamon Press: Oxford, 1991; Vol. 2, pp 865-892. doi:10.1016/B978-0-08-052349-1.00051-2

2. Roth, M.; Dubs, P.; Götschi, E.; Eschenmoser, A. Helv. Chim. Acta 1971, 54, 710-734. doi:10.1002/hlca.19710540229

3. Knott, E. B. J. Chem. Soc. 1955, 916-927. doi:10.1039/JR9550000916

4. Eschenmoser, A.; Wintner, C. E. Science 1977, 196, 1410-1426. doi:10.1126/science.867037

5. Ireland, R. E.; Brown, F. R., Jr. J. Org. Chem. 1980, 45, 1868-1880. doi:10.1021/jo01298a022

6. Neto, B. A. D.; Lapis, A. A. M.; Bernd, A. B.; Russowsky, D. Tetrahedron 2009, 65, 2484-2496. doi:10.1016/j.tet.2009.01.081

7. Wlodarczak, J.; Wysocka, W.; Katrusiak, A. J. Mol. Struct. 2010, 971, 12-17. doi:10.1016/j.molstruc.2010.02.050

8. Nakajima, R.; Ogino, T.; Yokoshima, S.; Fukuyama, T. J. Am. Chem. Soc. 2010, 132, 1236-1237. doi:10.1021/ja9103233

9. Elliott, M. C.; Long, M. S. Org. Biomol. Chem. 2004, 2, 2003-2011. doi:10.1039/b404679j

10. Pettersson, B.; Hasimbegovic, V.; Bergman, J. J. Org. Chem. 2011, 76, 1554-1561. doi:10.1021/jo101864n

11. Mulzer, J.; List, B.; Bats, J. W. J. Am. Chem. Soc. 1997, 119, 5512-5518. doi:10.1021/ja9700515

12. Russowsky, D.; da Silveira Neto, B. A. Tetrahedron Lett. 2003, 44, 2923-2926. doi:10.1016/s0040-4039(03)00420-9

13. Nicolaou, K. C.; Reddy, K. R.; Skokotas, G.; Sato, F.; Xiao, X. Y.; Hwang, C. K. J. Am. Chem. Soc. 1993, 115, 3558-3575. doi:10.1021/ja00062a021

14. Singh, S.; Schober, A.; Gebinoga, M.; Groß, G. A. Tetrahedron Lett. 2009, 50, 1838-1843. doi:10.1016/j.tetlet.2009.02.027

15. Groß, G. A.; Wurziger, H.; Schlingloff, G.; Schober, A. QSAR \& Comb. Sci. (Special Issue: Array Synthesis) 2006, 25, 1055-1062. doi:10.1002/qsar.200640120

16. Groß, G. A.; Mayer, G.; Albert, J.; Riester, D.; Osterodt, J.; Wurziger, H.; Schober, A. Angew. Chem., Int. Ed. 2006, 118, 3174-3178. doi:10.1002/ange.200503041

17. Gebinoga, M.; Groß, G. A.; Albrecht, A.; Lübeck, T.; Henkel, T.; Hoffmann, P.; Klemm, U.; Schlingloff, G.; Frank, T.; Schober, A. QSAR \& Comb. Sci. (Special Issue: Array Synthesis) 2006, 25, 1063-1068. doi:10.1002/qsar.200640113

18. Wegner, J.; Ceylan, S.; Kirschning, A. Chem. Commun. 2011, 47, 4583-4592. doi:10.1039/c0cc05060a

19. Singh, S.; Schober, A.; Gebinoga, M.; Groß, G. A. Tetrahedron Lett. 2011, 52, 3814-3817. doi:10.1016/j.tetlet.2011.05.067

\section{License and Terms}

This is an Open Access article under the terms of the Creative Commons Attribution License

(http://creativecommons.org/licenses/by/2.0), which permits unrestricted use, distribution, and reproduction in any medium, provided the original work is properly cited.

The license is subject to the Beilstein Journal of Organic Chemistry terms and conditions:

(http://www.beilstein-journals.org/bjoc)

The definitive version of this article is the electronic one which can be found at:

$\underline{\text { doi:10.3762/bjoc. } 7.135}$ 